\title{
TYPICAL CHARACTERISTICS OF CHANCE AND NON-CHANCE COMPACT GROUPS OF GALAXIES
}

\author{
LUDMILA KISELEVA ${ }^{1}$, JOANNA ANOSOVA ${ }^{2}$ \\ 1.Institute of Astronomy, University of Cambridge, Madingley \\ Road, Cambridge CB3 OHA, England. \\ 2.National Astronomical Observatory, Tokyo 181, Japan.
}

In our previous works (Anosova 1987, Anosova and Kiseleva 1993) we developed a new objective statistical method for an identification of members of star and galaxy clusters as chance or non-chance ones. In the case of galaxies this method uses simultaneously their radial velocities $V$ and angular separations $\rho$.

In this work, we examine the galaxy fields with various $V$ corresponding to the observed data for the CFA galaxies: $1000 \leq V \leq 45000 \mathrm{~km} / \mathrm{s}$.

We construct model pairs of galaxies with various values of angular separations $\rho$ and differences of radial velocities $d V$. Using our new method, we find the typical values for relative quantities $d V_{c h}$ and $\rho_{c h}$ for confident chance pairs of galaxies .

We shown that for small $V$, and correspondently, small $d V$, for typical chance pairs the value of $\left\langle\rho_{c h}\right\rangle$ is large. With increasing $V$ and $d V$ these values decrease quickly. For the largest $V(45000 \mathrm{~km} / \mathrm{s})\left\langle\rho_{c h}\right\rangle=$ $(3.0 \pm 1.2)^{\prime}$. For this value of $V,\left\langle d V_{c h}\right\rangle=(1289 \pm 1087) \mathrm{km} / \mathrm{s}$. Therefore, we can see that if the group of galaxies is very far from the Sun, the two dimensional projection may be compact, but velocity differences $d V$ may be more then $1000 \mathrm{~km} / \mathrm{s}$ for chance members. If $d V$ is much less than this, then this group is a physically connected one; if $d V$ is much more than $1000 \mathrm{~km} / \mathrm{s}$ then it is a confident non-chance phenomenon. It may be an effect of projection or the radial velocities of the galaxies are not indicative of their Hubble distances.

\section{References}

Anosova J.P.,1987,Astrofizika,27,535.

Anosova,J.P.,Kiseleva,L.G.1993,Astrophys.Space Sc.,209,181. 